\title{
Correction: Evaluation of copy-number variants as modifiers of breast and ovarian cancer risk for BRCA1 pathogenic variant carriers
}

\author{
Logan C. Walker ${ }^{1} \cdot$ Louise Marquart ${ }^{2}$ - John F. Pearson ${ }^{3} \cdot$ George A. R. Wiggins ${ }^{1} \cdot$ Tracy A. O'Mara $^{4}$. \\ Michael T. Parsons ${ }^{4} \cdot$ BCFR $^{5}$ - Daniel Barrowdale ${ }^{6}$. Lesley McGuffog ${ }^{6} \cdot$ Joe Dennis $^{6} \cdot$ Javier Benitez $^{7}$. \\ Thomas P. Slavin ${ }^{8} \cdot$ Paolo Radice $^{9} \cdot$ Debra Frost $^{6} \cdot$ EMBRACE $^{6} \cdot$ Andrew K. Godwin $^{10} \cdot$ Alfons Meind $^{11}$. \\ Rita Katharina Schmutzler ${ }^{12}$. GEMO Study Collaborators ${ }^{13,14}$. Claudine Isaacs ${ }^{15}$ - Beth N. Peshkin ${ }^{15}$. \\ Trinidad Caldes ${ }^{16}$. Frans B. L. Hogervorst ${ }^{17} \cdot$ HEBON $^{18} \cdot$ Conxi Lazaro $^{19}$ - Anna Jakubowska ${ }^{20}$. \\ Marco Montagna ${ }^{21} \cdot \mathrm{KConFab}_{\text {Investigators }}^{22,23} \cdot$ Xiaoqing Chen $^{4} \cdot$ Kenneth Offit $^{24}$ - Peter J. Hulick ${ }^{25}$. \\ Irene L. Andrulis ${ }^{26} \cdot$ Annika Lindblom $^{27} \cdot$ Robert L. Nussbaum ${ }^{28} \cdot$ Katherine L. Nathanson $^{29}$. \\ Georgia Chenevix-Trench ${ }^{4} \cdot$ Antonis C. Antoniou ${ }^{6} \cdot$ Fergus J. Couch $^{30} \cdot$ Amanda B. Spurdle $^{4}$
}

Published online: 22 August 2018

(c) The Author(s) 2018. This article is published with open access

Correction to: European Journal of Human Genetics https://doi.org/10.1038/ejhg.2016.203; Article published online 1 February 2017

1 Department of Pathology, University of Otago, Christchurch, New Zealand

2 Statistics Unit, QIMR Berghofer Medical Research Institute, Brisbane, Queensland, Australia

3 Biostatistics and Computational Biology Unit, Department of the Dean, University of Otago, Christchurch, New Zealand

4 Genetics and Computational Biology Division, QIMR Berghofer Medical Research Institute, Brisbane, Queensland, Australia

5 Department of Epidemiology, Cancer Prevention Institute of California, Fremont, CA, USA

6 Department of Public Health and Primary Care, Centre for Cancer Genetic Epidemiology, University of Cambridge, Strangeways Research Laboratory, Worts Causeway, Cambridge, UK

These authors contributed equally: Logan C. Walker, Louise Marquart. The original article can be found online at https://doi.org/10.1038/ejhg. 2016.203.

$$
\begin{aligned}
& \text { Logan C. Walker } \\
& \text { logan.walker@otago.ac.nz }
\end{aligned}
$$

Extended author information available on the last page of the article.

This Article was originally published under a CC BYNC-SA 4.0 license, but has now been made available under a CC BY 4.0 license. The PDF and HTML versions of the Article have been modified accordingly. Madrid, Spain

Department of Population Sciences Division of Clinical Cancer Genomics, City of Hope Clinical Cancer Genomics Community Research Network, Duarte, CA, USA

9 Department of Preventive and Predictive Medicine, Unit of Molecular Bases of Genetic Risk and Genetic Testing, Fondazione IRCCS (Istituto Di Ricovero e Cura a Carattere Scientifico) Istituto Nazionale Tumori (INT), Milan, Italy

10 Department of Pathology and Laboratory Medicine, University of Kansas Medical Center, Kansas City, KS, USA

11 Department of Gynaecology and Obstetrics, Division of Tumor Genetics, Klinikum rechts der Isar, Technical University Munich, Munich, Germany

12 Center for Hereditary Breast and Ovarian Cancer, Medical Faculty, University Hospital Cologne, Cologne, Germany

13 Department of Tumour Biology, Institut Curie, Paris, France

14 Institut Curie, INSERM, Paris, France

15 Lombardi Comprehensive Cancer Center, Georgetown University, Washington, USA

16 Molecular Oncology Laboratory CIBERONC, Hospital Clinico San Carlos, IdISSC (El Instituto de Investigación Sanitaria del Hospital Clínico San Carlos), Madrid, Spain 
17 Family Cancer Clinic, Netherlands Cancer Institute, Amsterdam, The Netherlands

18 The Hereditary Breast and Ovarian Cancer Research Group Netherlands (HEBON), Coordinating center: Netherlands Cancer Institute, Amsterdam, The Netherlands

19 Molecular Diagnostic Unit, Hereditary Cancer Program, IDIBELL (Bellvitge Biomedical Research Institute), Catalan Institute of Oncology, Gran Via de l'Hospitalet, Barcelona, Spain

20 Department of Genetics and Pathology, Pomeranian Medical University, Szczecin, Poland

21 Immunology and Molecular Oncology Unit, Veneto Institute of Oncology IOV - IRCCS, Padua, Italy

22 kConFab, Research Department, Peter MacCallum Cancer Centre, Melbourne, Australia

23 The Sir Peter MacCallum Department of Oncology University of Melbourne, Parkville, Australia

24 Department of Medicine, Cancer Biology and Genetics, Clinical Genetics Research Laboratory, Memorial Sloan-Kettering Cancer Center, New York, NY, USA

25 Center for Medical Genetics, NorthShore University HealthSystem, Evanston, IL, USA

26 Lunenfeld-Tanenbaum Research Institute, Mount Sinai Hospital,
Toronto, Ontario, Canada

27

Department of Clinical Genetics, Karolinska University Hospital, Stockholm, Sweden

28 Department of Medicine and Institute for Human Genetics, University of California, San Francisco, CA, USA

29 Department of Medicine and the Abramson Cancer Center, Perelman School of Medicine at the University of Pennsylvania, Philadelphia, PA, USA

30 Department of Laboratory Medicine and Pathology, and Health Sciences Research, Mayo Clinic, Rochester, MN, USA

Open Access This article is licensed under a Creative Commons Attribution 4.0 International License, which permits use, sharing, adaptation, distribution and reproduction in any medium or format, as long as you give appropriate credit to the original author(s) and the source, provide a link to the Creative Commons license, and indicate if changes were made. The images or other third party material in this article are included in the article's Creative Commons license, unless indicated otherwise in a credit line to the material. If material is not included in the article's Creative Commons license and your intended use is not permitted by statutory regulation or exceeds the permitted use, you will need to obtain permission directly from the copyright holder. To view a copy of this license, visit http://creativecommons. org/licenses/by/4.0/. 